\title{
LncRNA RBM5-AS1 Promotes Osteosarcoma Cell Proliferation, Migration, and Invasion
}

\author{
Biyong Deng, ${ }^{1}$ Runsang Pan, ${ }^{2}$ Xin Ou, ${ }^{1}$ Taizhe Wang, ${ }^{1}$ Weiguo Wang, ${ }^{3}$ Yingjie Nie $\mathbb{D},{ }^{4}$ \\ and Houping Chen $\mathbb{D}^{2}$ \\ ${ }^{1}$ The Orthopedic Surgery Department of Guizhou Province Orthopedic Hospital, China \\ ${ }^{2}$ The Orthopedic Surgery Department of Guiyang Maternal and Child Health-Care Hospital, Guiyang, \\ Guizhou Province 550000, China \\ ${ }^{3}$ Department of Pathology of Guizhou Province Orthopedic Hospital, China \\ ${ }^{4}$ Science and Education Division of Guizhou Province People's Hospital, Guiyang, Guizhou Province 550000, China
}

Correspondence should be addressed to Yingjie Nie; nienyj@hotmail.com and Houping Chen; chenhouping@126.com

Received 31 May 2020; Revised 28 January 2021; Accepted 23 February 2021; Published 16 March 2021

Academic Editor: K. H. Mok

Copyright (c) 2021 Biyong Deng et al. This is an open access article distributed under the Creative Commons Attribution License, which permits unrestricted use, distribution, and reproduction in any medium, provided the original work is properly cited.

Purpose. Osteosarcoma (Os) is the most frequent malignant tumor of the bone in the pediatric age group, and accumulating evidences show that lncRNAs play a key role in the development of Os. Thus, we investigated the role of RBM5-AS1 and its molecular mechanism. Methods. The expression of RBM5-AS1 in Os tissues and cell lines was detected by real-time polymerase chain reaction (QPCR). The effect of RBM5-AS1 on the proliferation of Os cells was detected using CCK8 assays and flow cytometry. The effect of RBM5-AS1 on the migration and invasion of Os cells was detected by transwell assays. And we performed QPCR and western blotting assays to investigate the relationship between RBM5-AS1 and RBM5. Finally, western blotting assays were performed to explore the mechanism of RBM5. Results. LncRNA RBM5-AS1 was overexpressed in the Os tissues and cell lines. And lncRNA RBM5-AS1 promoted Os cell proliferation, migration, and invasion in vitro and tumor growth in vivo. LncRNA RBM5-AS1 targets RBM5 in Os cells. Conclusion. To sum up, the results showed that lncRNA RBM5AS1 promotes cell proliferation, migration, and invasion in Os.

\section{Introduction}

Osteosarcoma (Os) is the most frequent malignant tumor of the bone in the pediatric age group [1], and the long-term survival rate for patients with localized Os is about $65 \%$, whereas it is less than $20 \%$ for patients with metastatic Os [2]. The outcome in survival rates highlights the need for novel pathways and targets [3].

Long noncoding RNA (lncRNA) is a large class of RNA molecules with size larger than 200 nucleotides and without protein-coding capability [4]. The functions of lncRNAs range broadly from regulating chromatin structure and gene expression in the nucleus to control messenger RNA (mRNA) processing, mRNA posttranscriptional regulation, cellular signaling, and protein activity in the cytoplasm [5].
With growing numbers of lncRNAs being assigned to biological functions, the specificity of the lncRNA expression is recognized as biomarkers and the development of highly targeted therapeutics [6].

RBM5-AS1 is a nuclear-retained transcript that selectively interacted with $\beta$-catenin [7]. Mechanistic investigations showed that RBM5-AS1 activity is critical for the functional enablement of colon cancer stem-like cells [8], but its function in Os is still unclear. So, we investigated the RBM5-AS1 molecular mechanisms.

Then, we predicted that RBM5-AS1 regulates RBM5. RBM5 (previously referred to as g15, LUCA-15, and H37) is an RNA-binding protein that has the ability to modulate apoptosis [9]. RBM5 is a tumor suppressor gene, and it has been found that it is most frequently deleted at the earliest 


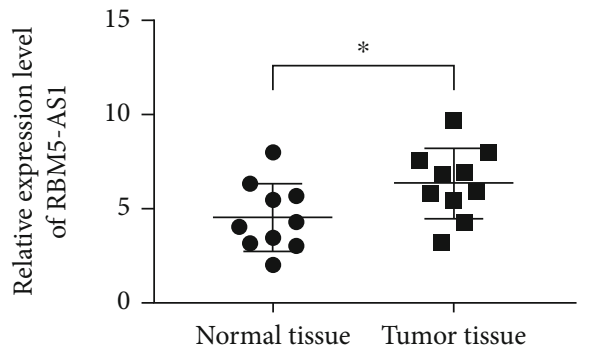

(a)

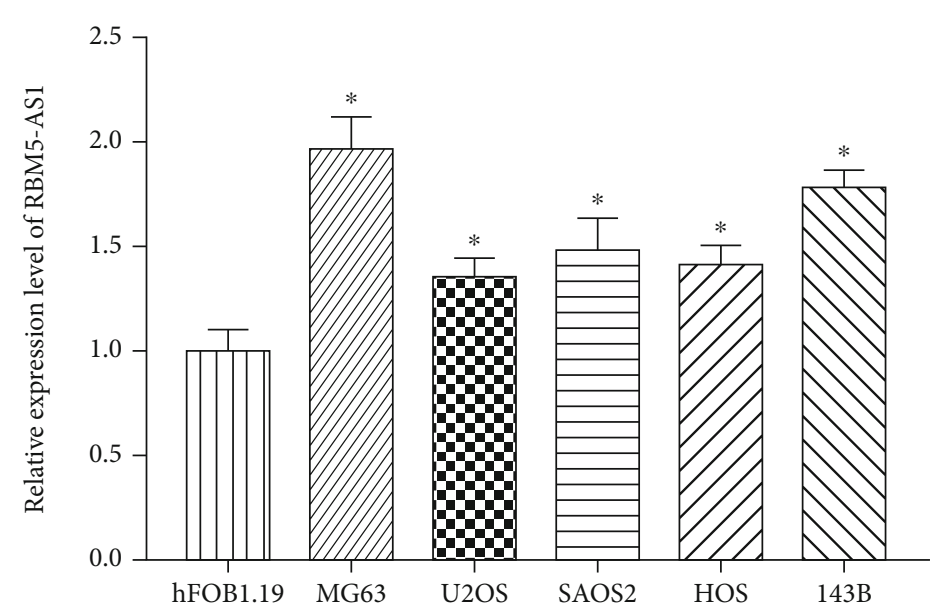

(b)

FIGURE 1: LncRNA RBM5-AS1 was overexpressed in the Os tissues and cell lines. (a) lncRNA RBM5-AS1 was significantly upregulated in Os tissues compared with normal tissues. (b) lncRNA RBM5-AS1 was significantly upregulated in Os cell lines compared with hFOB1.19 cells by RT-PCR. ${ }^{*} P<0.05$.

stage of lung cancer development $[10,11]$. And in this article, we study the effect of RBM5-AS1 to RBM5.

\section{Materials and Methods}

2.1. Patients and Cell Lines. Os samples were acquired from the Department of Orthopedic Surgery, Guizhou Province People's Hospital (Guiyang, China). The study protocol was approved by the Animal Care Welfare Committee of the Guizhou Province Orthopedic Hospital. The human approval number is No. 20190706032. The human Os cell lines MG63, U2OS, SAOS2, HOS, 143B, and the normal osteoblast cell line hFOB1.19 were acquired from ATCC (American Type Culture Collection; Manassas, VA, USA). All cell lines were cultured at $37^{\circ} \mathrm{C}$ in an atmosphere containing $5 \% \mathrm{CO}_{2}$ in DMEM or RPMI-1640 (Gibco, New York, USA) supplemented with $10 \%$ fetal bovine serum (Thermo Fisher Scientific, Massachusetts, USA).

2.2. Cell Transfection. Cells $\left(2 \times 10^{5}\right)$ were seeded on the day before transfection with shRNAs (RiboBio, Guangzhou, China). Transfection was performed using lipofectamine 3000 (Life Technologies Co., Carlsbad, CA, USA) according to the manufacturer's instructions. We performed assays after $48 \mathrm{~h}$. The sequence of RBM5-AS1 NC shRNA is $5^{\prime}$ -GATTCTTCTTCTGTTCTGACATACT- ${ }^{\prime}$, and the sequence of RBM5-AS1 shRNA is $5^{\prime}$-CCTTTCATTCT GAATTCATGTGCTT-3' . RBM5 upregulated and downregulated lentiviruses were purchased from GeneChem (Shanghai, China). All transfections were performed according to the manufacturer's instructions.

2.3. RNA Isolation, Reverse Transcription, and QPCR. Total RNA were extracted using TRIzol (Invitrogen, Carlsbad, CA, USA) reagent according to the manufacturer's instructions. Reverse transcription of lncRNAs was performed with PrimeScript RT Master Mix (Takara, Japan). QPCR was conducted using SYBR Green (Takara, Japan) according to the manufacturer's instructions. The results were normalized to the GADPH expression and calculated according to the $2^{-\triangle \Delta \mathrm{Cq}}$ method. All experiments were in triplicate. Primer sequences were showed as follows: RBM5-AS1 forward sequence: 5' -TGGGAATGGGGAAGAGAAC-3, RBM5AS1 reversed sequence: $5^{\prime}$-GGGAATAGTGTGTGGCAAA AG-3'; RBM5 forward sequence: $5^{\prime}$-GCACGACTATA GGCATGACAT-3', RBM5 reversed sequence: $5^{\prime}$-AGTC AAACTTGTCTGCTCCA-3'.

2.4. Western Blotting. Total cell proteins were extracted using RIPA lysis buffer (Solabio, Beijing, China) containing protease and phosphatase inhibitors (Roche, Basel, Switzerland). Proteins $(20 \mu \mathrm{g})$ were resolved using $10 \%$ SDS polyacrylamide electrophoresis and electrotransferred to polyvinylidene difluoride (PVDF) membrane (Millipore, Bedford, MA). Western blots were probed with antibodies against RMB5 and GAPDH $\left(1: 1000\right.$, CST, USA) at $4^{\circ} \mathrm{C}$ overnight. After washing, the blots were then incubated with the secondary antibodies, goat anti-mouse (1:2000), and goat anti-rabbit $(1: 2000)$ (Boster Bio, USA) for $2 \mathrm{~h}$ at room temperature.

2.5. Colony Formation Assay. Transfected Os cells were planted into 6 -well plates $\left(1 \times 10^{3}\right.$ cells/plate $)$ and incubated in DMEM or RPMI 1640 supplemented with 10\% FBS at $37^{\circ} \mathrm{C}$ for 2 weeks. Then, cells were fixed in methanol for 30 minutes, stained with $1 \%$ crystal violet dye, and then counted. All experiments were performed with three independent trials.

2.6. Flow Cytometry. Transfected Os cells were planted into 6-well plates and cultured 2 days, and MG63 and 143B cells were fixed with $70 \%$ ethanol, respectively, and signed with propidium iodide (Sigma-Aldrich) in the dark $(50 \mathrm{~g} / \mathrm{mL})$ for about 30 minutes in the presence of RNaseA (SigmaAldrich). And samples were detected by flow cytometry (Beckman, Cytomics FC 500, USA). 


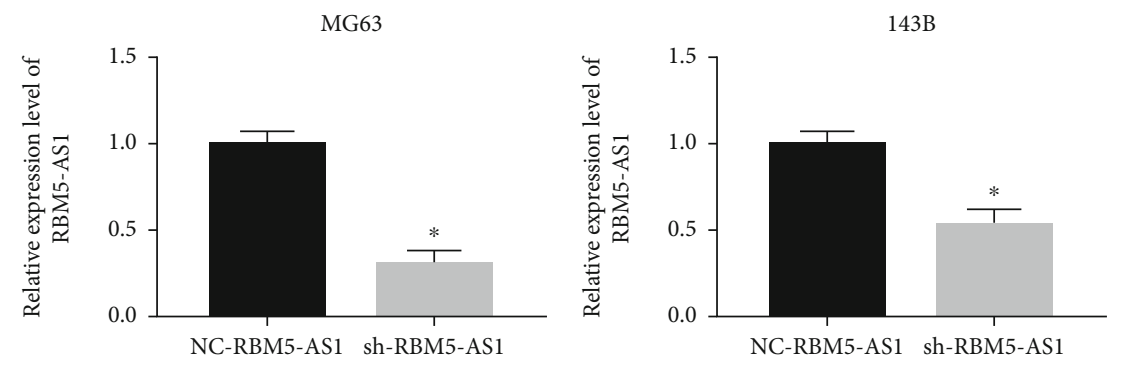

(a)

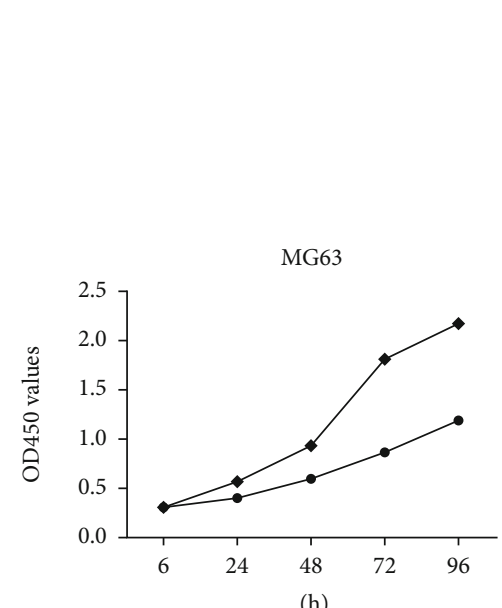

(h)

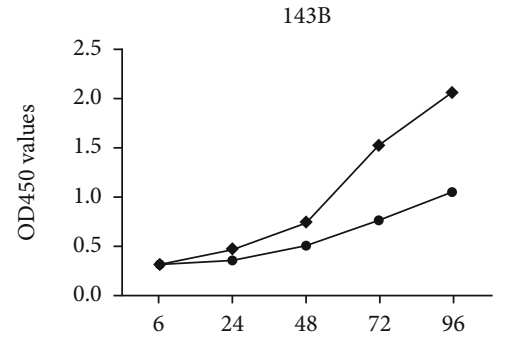

(h)

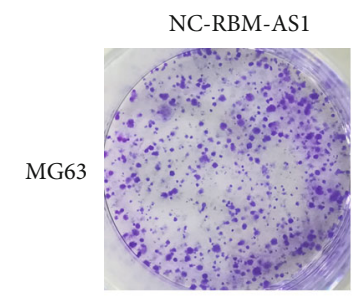

sh-RBM5-AS1

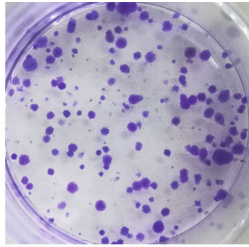

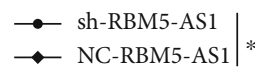

(b)
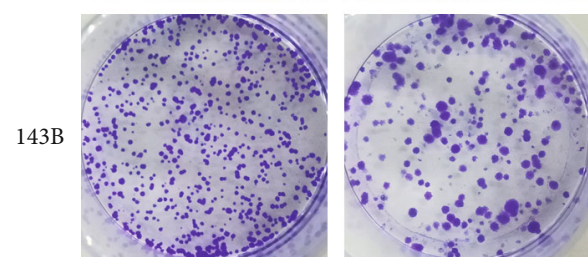

(c)

NC-RBM5-AS1

sh-RBM5-AS1

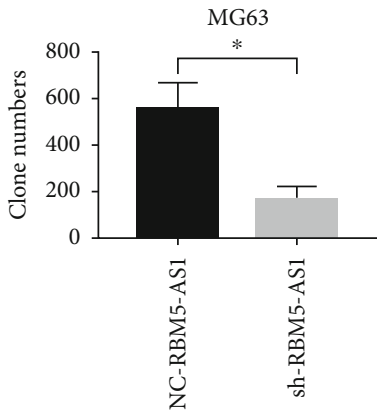

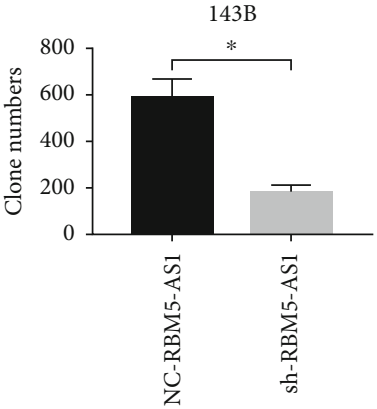

(d)
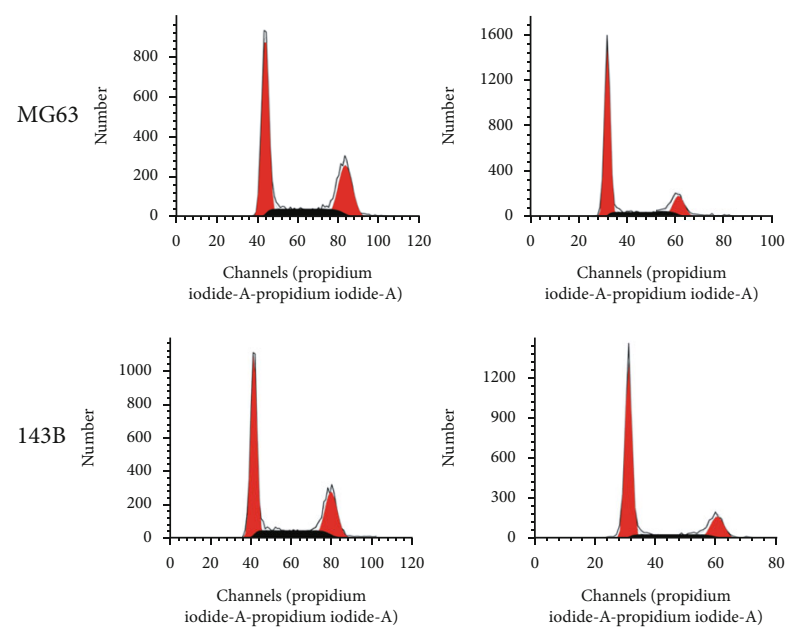

(e)

Figure 2: Continued. 


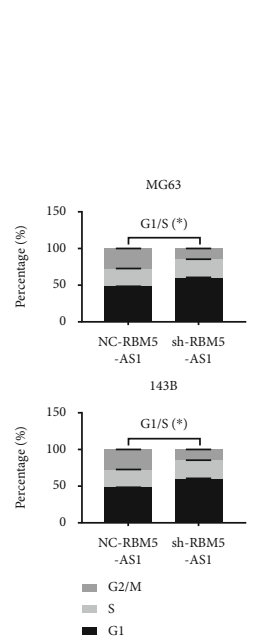

(f)

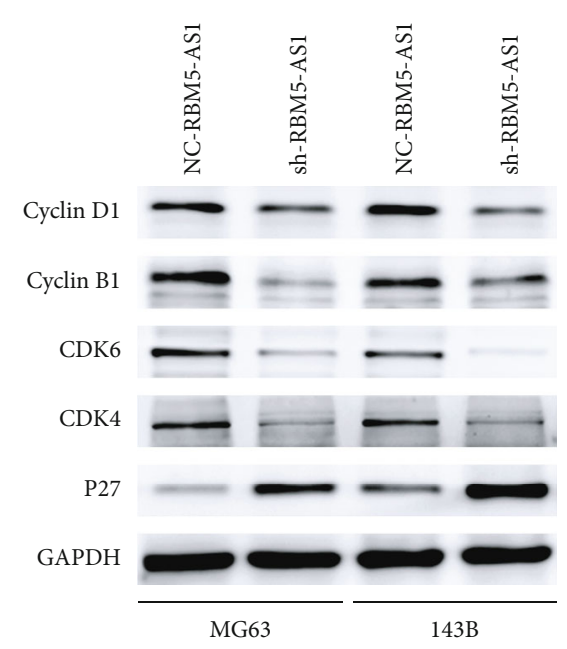

(g)

FIGURE 2: LncRNA RBM5-AS1 promoted Os cells proliferation in vitro. (a) Stable lncRNA RBM5-AS1 knockdown in MG63 and 143B cells was confirmed by RT-PCR. (b) CCK8 assays showed the effect of RBM5-AS1 on the proliferation of MG63 and 143B cells. (c, d) Colony formation assays showed a significantly lower proliferative rate in both cell lines following RBM5-AS1 knockdown. (e, f) Flow cytometry was used to explore whether RBM5-AS1 promoted Os cell proliferation. (g) Western blotting was used to detect the protein level of cyclin B1, cyclin D1, CDK4, and CDK6 after RBM5-AS1 inhibition.

2.7. Xenograft Transplantation. Four weeks female nude mice (Beijing Huayi Kang Company, Beijing, China) were divided into two groups, three per group. MG63 cells were divided into NC and sh-RMB5-AS1 groups, $1 \times 10^{6}$ of each was intraperitoneally injected, and the mice were observed each week and weighed. After 8 weeks, or when the nude mice died naturally, the surviving mice were killed using cervical dislocation, and tumors were removed and weighed. The animal ethical approval number is No. 20000584.

2.8. Transwell Migration and Invation Assays. Transwell invation assays were performed by transwell chambers (Corning- Costar; pore size $8 \mu \mathrm{m}$ ) coated with Matrigel (Sigma); though, migration assays were without Matrigel. Transfected Os cells resuspended with FBS-free medium were added to the top chamber, and the medium with $10 \%$ FBS was filled in the bottom chamber as chemotaxin. After $48 \mathrm{~h}$, we fixed and stained the cells. Cells from five random fields ( $\times 40$ magnifications) were counted and photographed under the microscope.

2.9. Statistical Analysis. The Student $t$-test was performed to analyze the significance of differences between the mean values of three independent experiments. A significant difference was defined as $P<0.05$. Results are displayed as mean \pm SD.

\section{Results}

3.1. LncRNA RBM5-AS1 Was Overexpressed in the Os Tissues and Cell Lines. To investigate the role of LncRNA RBM5-AS1 in the Os, the expression of RBM5-AS1 between normal tissues and Os tissues was detected. The results showed that the expression of LncRNA RBM5-AS1 was significantly upregulated in the Os tissues compared with normal tissues
(Figure 1(a), $P<0.05)$. And the expression among different Os cell lines and normal cells was also detected through QPCR. As shown in Figure 1(b), the results made significantly differences, and the expression of RBM5-AS1 was overexpressed in the Os cells.

3.2. Downregulated IncRNA RBM5-AS1 Inhibited Os Cells Proliferation In Vitro. To investigate the function of lncRNA RBM5-AS1 in Os cells, the RBM5-AS1 was downregulated by transfecting lentivirus. And the cells were divided into the negative control (NC-RBM5-AS1) group and sh-RBM5AS1 group. As shown in Figure 2(a), the expression of RBM5-AS1 in the sh-RBM5-AS1 group was downregulated. And CCK8 results showed that downregulated RBM5-AS1 inhibited the Os cell proliferation (Figure 2(b)). And the colony formation assays displayed sh-RBM5-AS1 groups reduced the capability of colony formation (Figures 2(c) and 2(d)). Then, we explored how lncRNA RBM5-AS1 effect the cell cycle by flow cytometry, and the results indicated that the fraction of cells in G1 phase increased, while the fraction of cells in the $S$ phase decreased compared with the NCRBM5-AS1 group (Figures 2(e) and 2(f)). Western blot analysis showed that sh-RBM5-AS1 groups reduced cyclin B1, cyclin D1, CDK4, and CDK6 which indicated that downregulated RBM5-AS1 promoted cell cycle progression (Figure 2(g)).

3.3. Downregulated IncRNA RBM5-AS1 Inhibited Os Cells Migration and Invasion In Vitro. We also performed the transwell assays to detect the ability of migration and invasion. The results showed that the migration and invasion cells in the NC-RBM5-AS1 groups were larger compared to the sh-RBM5-AS1 groups (Figures 3(a)-3(d)). 


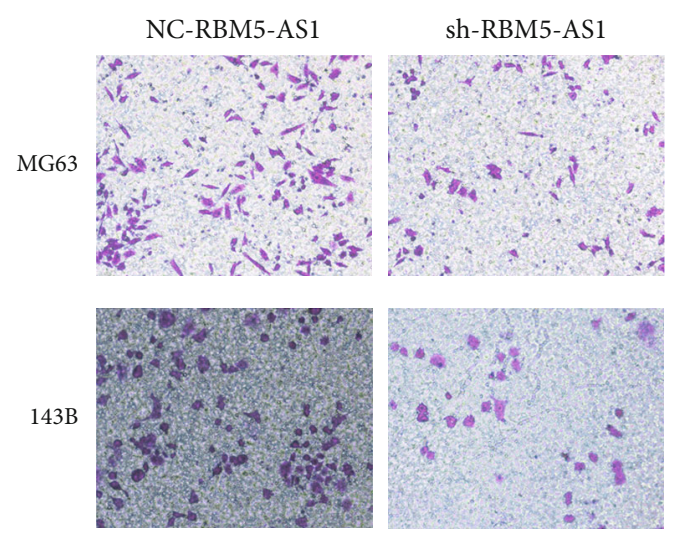

(a)

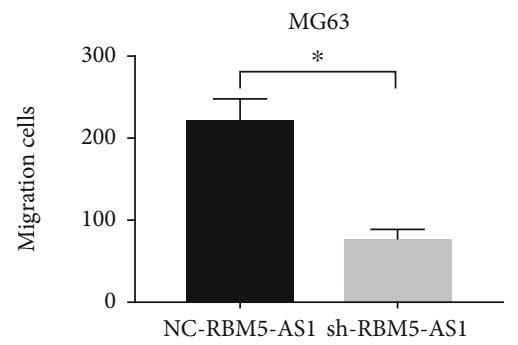

(b)

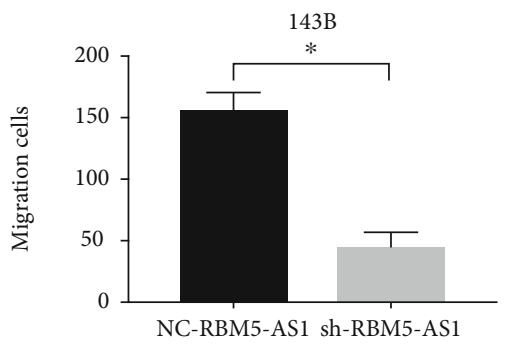

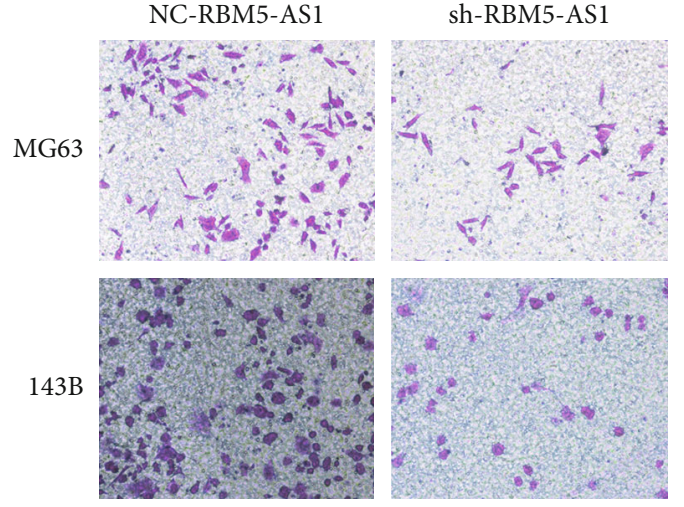

(c)
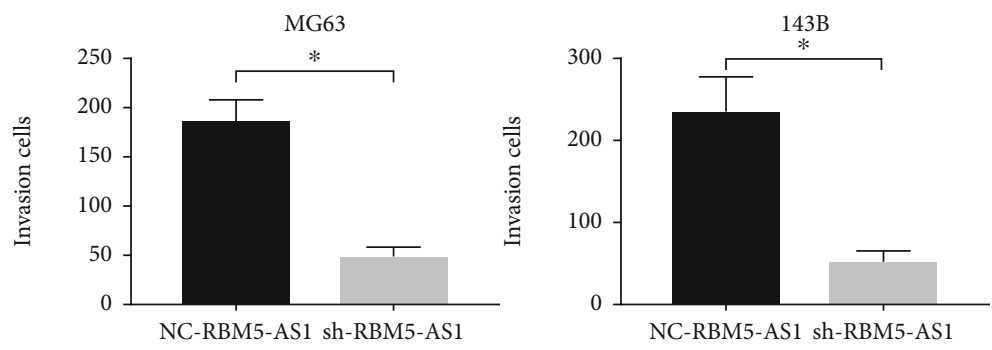

(d)

Figure 3: LncRNA RBM5-AS1 promoted Os cell migration and invasion in vitro. (a, b) Transwell assays showed significantly decreased migration abilities in MG63 and 143B cells while RBM5-AS1 knockdown. (c, d) Transwell assays showed significantly decreased invasion abilities in both cell lines while RBM5-AS1 knockdown. ${ }^{*} P<0.05$.

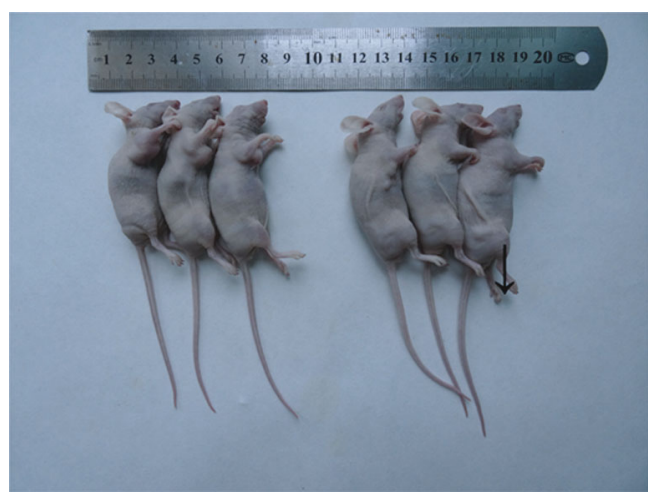

(a)

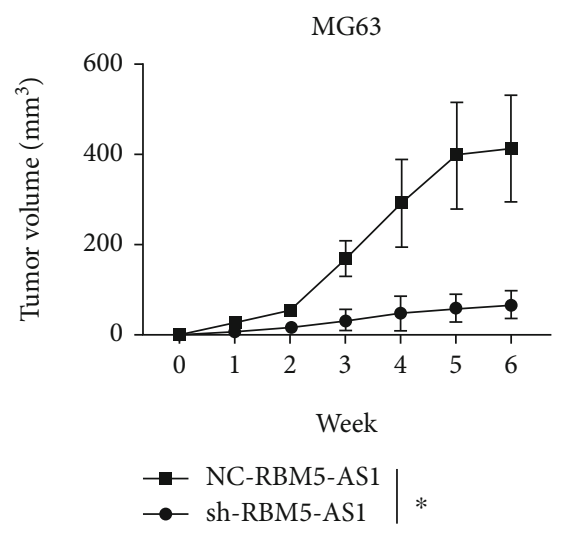

(b)

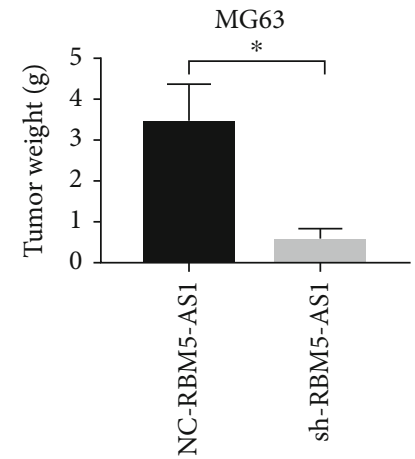

(c)

FIGURE 4: IncRNA RBM5-AS1 promoted Os tumor growth in vivo. (a) The results showed that lncRNA RBM5-AS1 promoted Os cell tumor growth in vivo. (b, c) The RBM5-AS1 knockdown group showed reduced tumor volumes and weight compared with controls. ${ }^{*} P<0.05$. 


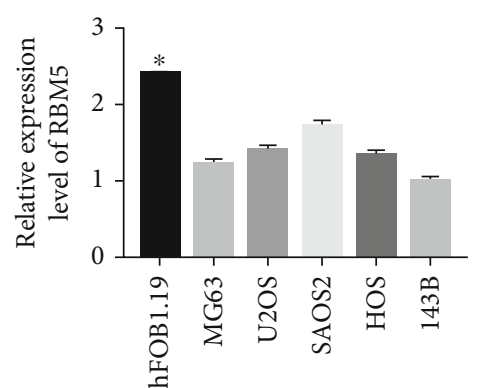

(a)

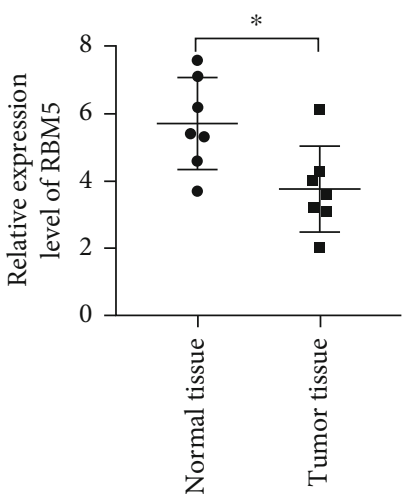

(b)
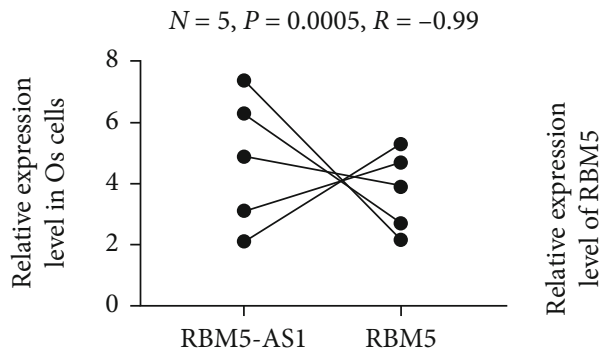

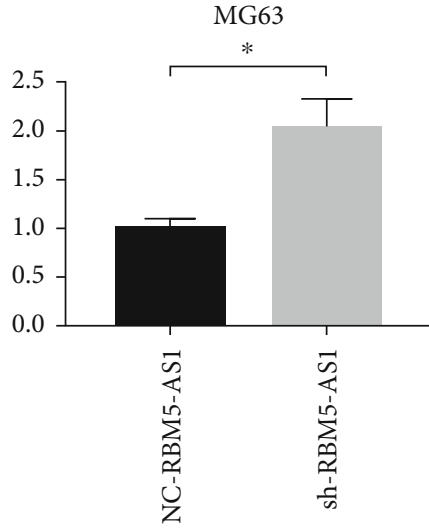

(d)

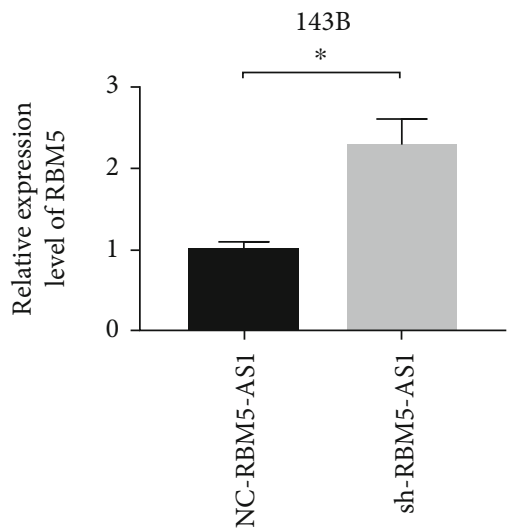

(c)

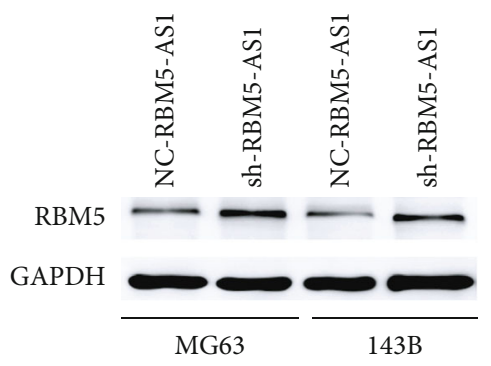

(e)
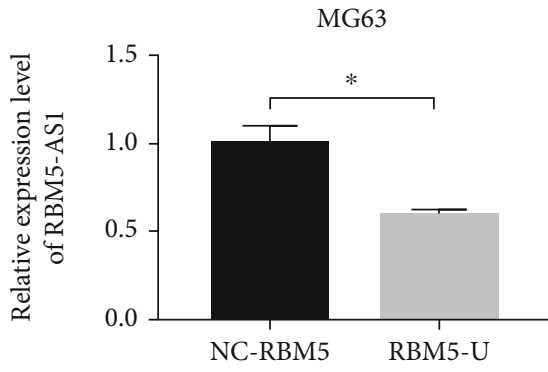

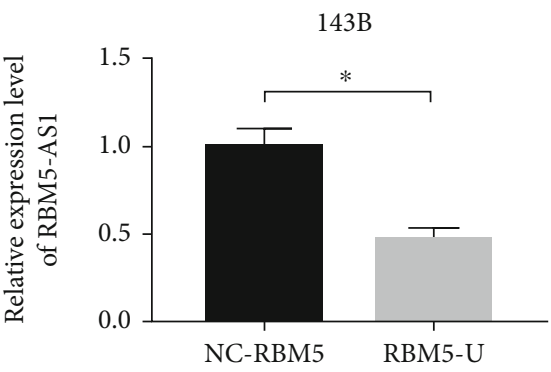

(f)

FIGURE 5: lncRNA RBM5-AS1 targets RBM5 in Os. (a, b) RT-PCR showed that the RBM5 expression was markedly reduced in Os cell lines and tissues. (c) The correlation between the RBM5-AS1 and RBM5 expression in Os cells. (d, e) The results showed that the RBM5 expression was markedly reduced in NC- RBM5-AS1 cells by RT-PCR and western blot. (f) The results showed that the RBM5-AS1 expression was markedly reduced in RBM5-U cells by RT-PCR.

3.4. Downregulated IncRNA RBM5-AS1 Inhibited Os Tumor Growth In Vivo. We established subcutaneous xenograft models to explore the effect of lncRNA RBM5-AS1. And the tumors in NC- RBM5-AS1 groups were significantly larger than that in the sh-RBM5-AS1 group (Figure 4(a)). In addition, the tumor volume and weight decreased significantly in the sh-RBM5-AS1 group (Figures 4(b) and 4(c)).

3.5. IncRNA RBM5-AS1 Targets RBM5 in Os Cells. Since lncRNA RBM5-AS1 is an antisense lncRNA of RBM5, we further investigated if RBM5 is a functional target of lncRNA RBM5-AS1. The relative expression of RBM5 in Os cell lines and tissues was detected by Qpcr, and the results showed that
RBM5 was downregulated (Figures 5(a) and 5(b)). And Figure 5(c) shows an opposite relationship between lncRNA RBM5-AS1 and RBM5 expression in Os cell lines. To further explore the interaction between lncRNA RBM5-AS1 and RBM5, we inhibited the expression of lncRNA RBM5-AS1, and the results showed that the expression of RBM5 was increased (Figures 5(d) and 5(e)). Next, we upregulated the expression of RBM5, and the expression of lncRNA RBM5AS1 was decreased (Figure 5(f)).

3.6. The Downregulated RBM5 Expression Could Recover the Inhibition of RBM5-AS1 Knockdown in Os Cell Lines. RBM5AS1 was overexpressed in Os tissues and cells, but RBM5 was 


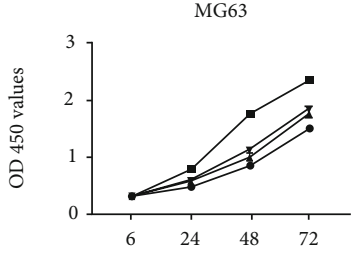

(h)

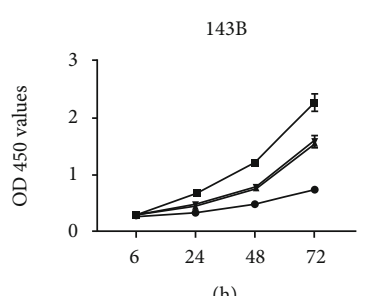

(h)

$\underset{-}{-}$ NC-RBM5-AS1
- sh-RBM5-AS1
- RBM5-D
$\longrightarrow$ -
sh-RBM5-AS1+RBM5-D

(a)

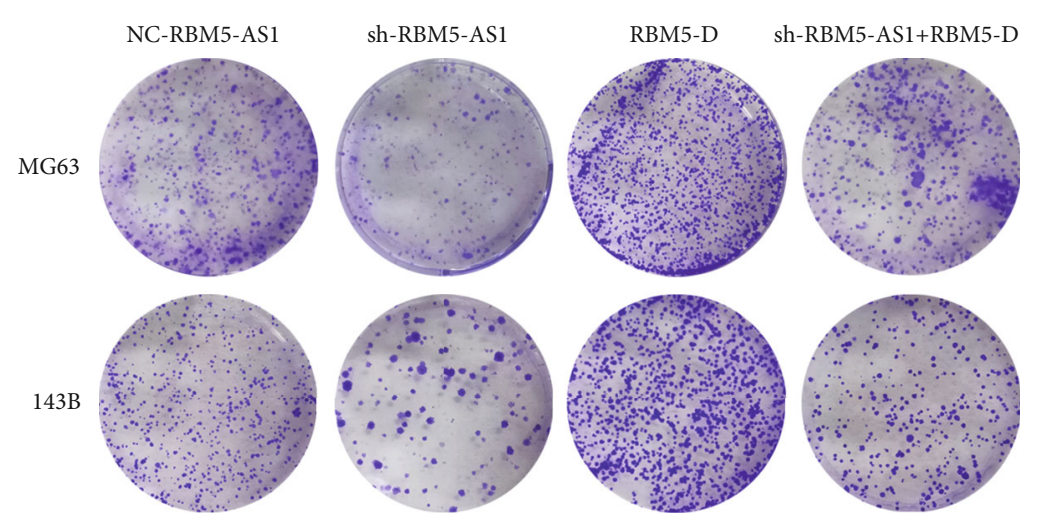

(b)

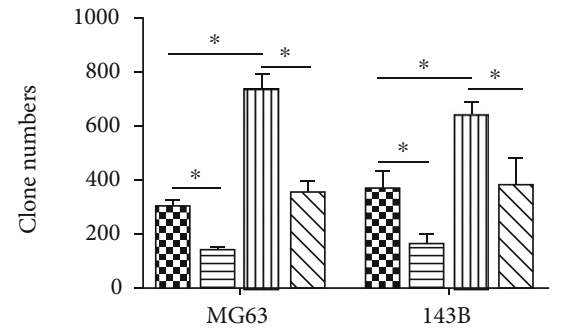

No NC-RBM5-AS1

○h-RBM5-AS1

IIII RBM5-D

$\square$ sh-RBM5-AS1+RBM5-D

(c)

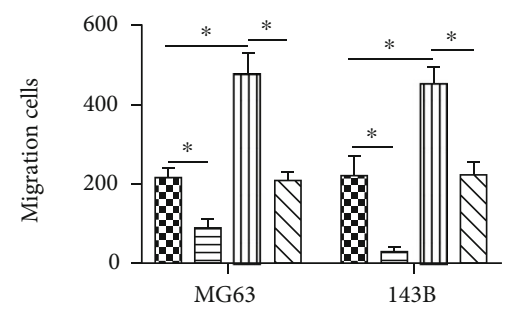

NC-RBM5-AS1

sh-RBM5-AS1

IIII RBM5-D

$\square$ sh-RBM5-AS1+RBM5-D

(e)

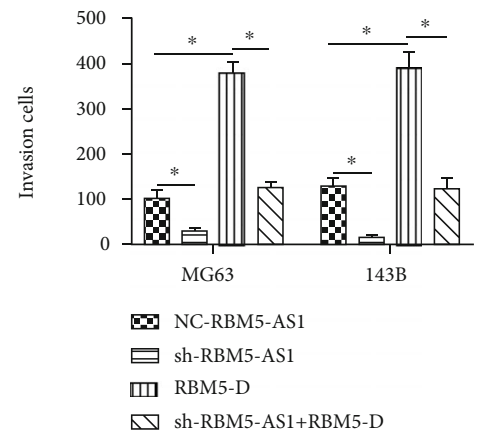

(g)

FIgURE 6: The downregulated RBM5 expression could recover the inhibition of RBM5-AS1 knockdown in Os cell lines. (a) The CCK8 assay was used to detect the proliferation of the NC-RBM5-AS1 group, sh-RBM5-AS1 group, RBM5-D group, and sh-RBM5-AS1+RBM5-D group, and the results showed that cells in the sh-RBM5-AS1+RBM5-D group rescued the inhibitory effect of sh-RBM5-AS1. (b, c) Cells in the shRBM5-AS1+RBM5-D group rescued the inhibitory effect of sh-RBM5-AS1 on colony formation. (d)-(g) Cells in the sh-RBM5-AS1+RBM5D group rescued the inhibitory effect of sh-RBM5-AS1 on Os cell migration and invasion. 
downregulated. To verify the function of RBM5 in Os progression, the Os cells were divided into the NC-RBM5-AS1 group, sh-RBM5-AS1 group, RBM5 downregulated group (RBM5-D group), and sh-RBM5-AS1+RBM5-D group. As the results showed, the sh-RBM5-AS1+ RBM5-D group rescued the inhibition of sh-RBM5-AS1 on cell proliferation (Figure 6(a)) and colony formation (Figures 6(b) and 6(c)). These results indicated that the downregulated RBM5 expression could recover the inhibition of RBM5-AS1 in Os cell lines. Furthermore, transwell assays indicated that the sh-RBM5-AS1+ RBM5$\mathrm{D}$ group rescued the inhibition of sh-RBM5-AS1 on cell migration and invasion (Figures 6(d) $-6(\mathrm{~g})$ ).

\section{Discussion}

Os is the most frequent malignant tumor of the bone in the pediatric age group, and the long-term survival rate for patients with localized Os is about $65 \%$, whereas it is less than $20 \%$ for patients with metastatic Os.

Accumulating evidence suggests that long noncoding RNA plays crucial roles in the progression of various cancers including regulation of gene expression, imprinting, chromatin modification, transcription, and posttranslational processing $[8,12]$. Li et al. generalized that many lncRNAs were important regulators for malignancies [13]. Zhou et al. investigated that lncRNA SNHG12 promoted tumorigenesis and metastasis in Os [14]; He et al. found that the lncRNA LINC00628 overexpression inhibited the growth and invasion through regulating the PI3K/Akt signaling pathway in Os [15]. In this article, we explored the effect of RBM5-AS1 in Os. Until now, there were few researches of RBM5-AS1 [8]. There has been reported that RBM5-AS1 participated in fracture healing and inhibited apoptosis of bone cells through the upregulation of $\beta$-catenin [7]. Li et al. studied that long noncoding RNA RBM5-AS1 promoted the aggressive behaviors of oral squamous cell carcinoma by regulating the miR1285-3p/YAP1 axis [16]. In the present study, we demonstrated that RBM5-AS1 was significantly increased in Os tissues and cell lines. In addition, knockdown of RBM5-AS1 significantly inhibited proliferation, migration, and invasion of Os cells in vitro as well as tumor growth in vivo.

Therefore, these results indicated that RBM5-AS1 promoted the progression of Os. And we predicted that RBM5AS1 acted on RBM5 because RBM5-AS1 was the antisense lncrna of RBM5. RBM5, an RNA-binding protein, was reported as a tumor suppressor gene. Jiang et al. investigated that RBM5 inhibited tumorigenesis of gliomas through inhibition of $\mathrm{Wnt} / \beta$-catenin signaling and induction of apoptosis [17]. Shao et al. reported that the tumor suppressor gene RBM5 inhibited lung adenocarcinoma cell growth and induced apoptosis [18]. And we found that RBM5 can rescue the inhibitory effect of RBM5-AS1 on Os cells. These results showed that RBM5-AS1 targeted RBM5, but the underlying mechanism is still unclear, and we can further research it.

\section{Data Availability}

The data used to support the findings of this study are available within the article.

\section{Ethical Approval}

This research was approved by the Human Research Ethics Committee of Guizhou Province People's Hospital and was conducted in accordance with the principles of the Declaration of Helsinki. All patients gave written, informed consent to participate in the study. All animal experiments were approved by the Guizhou Province People's Hospital on the Ethics of Animal Experiments (IRB). All treatments were in accordance with the US Public Health Service Policy on Humane Care and Use of Laboratory Animals.

\section{Conflicts of Interest}

The authors declare that they have no conflicts of interests.

\section{Authors' Contributions}

Biyong Deng, Runsang Pan, and Xin Ou contributed equally to this study.

\section{Acknowledgments}

We thank the Department of Orthopedic Surgery, Guizhou Province Orthopedic Hospital (Guiyang, China). The Os tissue samples and related anonymous clinical data were from The Guizhou Province People's Hospital. This study was funded by grants from the Department of Science and Technology of Guizhou ([2017]1093) and the Department of Science and Technology of Guiyang ([2018]1-64).

\section{References}

[1] R. Zamborsky, M. Kokavec, S. Harsanyi, and L. Danisovic, "Identification of Prognostic and Predictive Osteosarcoma Biomarkers," Medical Sciences, vol. 7, no. 2, p. 28, 2019.

[2] S. Miwa, T. Shirai, N. Yamamoto et al., "Current and emerging targets in immunotherapy for osteosarcoma," Journal of Oncology, vol. 2019, Article ID 7035045, 8 pages, 2019.

[3] D. J. Harrison, D. S. Geller, J. D. Gill, V. O. Lewis, and R. Gorlick, "Current and future therapeutic approaches for osteosarcoma," Expert Review of Anticancer Therapy, vol. 18, no. 1, pp. 39-50, 2018.

[4] C. H. Li and Y. Chen, "Insight into the role of long noncoding RNA in cancer development and progression," International Review of Cell and Molecular Biology, vol. 326, pp. 33-65, 2016.

[5] M. L. Luo, "Methods to study long noncoding RNA biology in cancer," Advances in Experimental Medicine and Biology, vol. 927, pp. 69-107, 2016.

[6] B. S. Gloss and M. E. Dinger, "The specificity of long noncoding RNA expression," Biochimica et Biophysica Acta, vol. 1859, no. 1, pp. 16-22, 2016.

[7] Y. Z. Huang, L. Zhao, C. L. Wang, S. J. Tian, S. Liu, and J. F. Ge, "RBM5-AS1 participates in fracture healing and inhibits apoptosis of bone cells through the up-regulation of $\beta$-catenin," European Review for Medical and Pharmacological Sciences, vol. 22, no. 16, pp. 5091-5097, 2018.

[8] S. Di Cecilia, F. Zhang, A. Sancho et al., "RBM5-AS1 is critical for self-renewal of colon cancer stem-like cells," Cancer Research, vol. 76, no. 19, pp. 5615-5627, 2016. 
[9] L. C. Sutherland, K. Wang, and A. G. Robinson, "RBM5 as a putative tumor suppressor gene for lung cancer," Journal of Thoracic Oncology, vol. 5, no. 3, pp. 294-298, 2010.

[10] V. V. Prabhu and N. Devaraj, "Regulating RNA binding motif 5 gene expression- a novel therapeutic target for lung cancer," Journal of Environmental Pathology, Toxicology and Oncology, vol. 36, no. 2, pp. 99-105, 2017.

[11] Y. Xu, Z. Su, J. Li et al., "Role of RNA-binding protein 5 in the diagnosis and chemotherapeutic response of lung cancer," Oncology Letters, vol. 17, no. 2, pp. 2013-2019, 2019.

[12] M. Isin and N. Dalay, "LncRNAs and neoplasia," Clinica Chimica Acta, vol. 444, pp. 280-288, 2015.

[13] Z. Li, P. Dou, T. Liu, and S. He, "Application of long noncoding RNAs in osteosarcoma: biomarkers and therapeutic targets," Cellular Physiology and Biochemistry, vol. 42, no. 4, pp. 1407-1419, 2017.

[14] S. Zhou, L. Yu, M. Xiong, and G. Dai, "LncRNA SNHG12 promotes tumorigenesis and metastasis in osteosarcoma by upregulating Notch2 by sponging miR-195-5p," Biochemical and Biophysical Research Communications, vol. 495, no. 2, pp. 1822-1832, 2018.

[15] Z. Chen, Y. Ma, Y. Pan, H. Zhu, C. Yu, and C. Sun, "MiR-1297 suppresses pancreatic cancer cell proliferation and metastasis by targeting MTDH," Molecular and Cellular Probes, vol. 40, pp. 19-26, 2018.

[16] C. Li, J. Ye, Z. Zhang, Z. Gong, Z. Lin, and M. Ding, "Long non-coding RNA RBM5-AS1 promotes the aggressive behaviors of oral squamous cell carcinoma by regulation of miR1285-3p/YAP1 axis," Biomedicine \& Pharmacotherapy, vol. 123, article 109723, 2020.

[17] Y. Jiang, H. Sheng, L. Meng et al., "RBM5 inhibits tumorigenesis of gliomas through inhibition of Wnt/ $\beta$-catenin signaling and induction of apoptosis," World Journal of Surgical Oncology, vol. 15, no. 1, p. 9, 2017.

[18] C. Shao, L. Zhao, K. Wang, W. Xu, J. Zhang, and B. Yang, "The tumor suppressor gene RBM5 inhibits lung adenocarcinoma cell growth and induces apoptosis," World Journal of Surgical Oncology, vol. 10, no. 1, p. 160, 2012. 